\title{
Sorption studies and characterization of developed biochar composites for As(III) adsorption from water
}

\author{
P. Singh \& D. Mohan \\ School of Environmental Sciences, Jawaharlal Nehru University, New Delhi, India
}

\begin{abstract}
Arsenic in groundwater is a serious problem. This paper investigates the role of biochar derived from agricultural waste byproducts to remediate arsenic from water. Composites were synthesized by thermal and chemical modifications in biomass. The biochar composites were characterized for their BET surface area and porosity. XPS, SEM, TEM, XRD, FT-IR, PPMS, ICP-MS were studied to analyze their magnetic moment, surface chemistry, mineralogy, crystallinity, elemental composition and functional group identification. Batch sorption studies, sorption equilibrium and kinetic studies were conducted using various mathematical equations and isotherm models to find sorption efficiency. As(III) removal occurred in $\mathrm{pH}$ 7.5-9.0. High adsorption capacities were reported for rice husk and wheat husk composites. Therefore, it can be considered to replace commercial adsorbents. Plausible mechanism and chemistry of As(III) adsorption was thus established.
\end{abstract}

\section{INTRODUCTION}

Arsenic is one of the most toxic contaminants in water. WHO has reported arsenic contamination in drinking water to have caused greatest number of deaths as compared to other heavy metals. WHO's permissible limit for arsenic in drinking water is $0.01 \mathrm{mg} \mathrm{L}^{-1}$. In India, states of West Bengal, Jharkhand, Bihar, Uttar Pradesh, Assam, Manipur and Chhattisgarh are reported to be most affected (Bhattacharya et al., 2011; Singh et al., 2015). Long term exposure is fatal to humans. Thus, removal of arsenic has become subject of great importance and attention. Out of many existing forms of As, $\mathrm{As}(\mathrm{III})$ is more toxic than $\mathrm{As}(\mathrm{V})$. Thus, low cost, simple performing techniques and methods have been used to obtain materials with high As removal efficiency $(\mathrm{Hu}$ et al., 2015; Ming et al., 2013; Mohan, 2007; Sun et al., 2015).

\section{METHODS/EXPERIMENTAL}

\subsection{Material}

Adsorption technique because of its simplicity and cost effectiveness has been used to remove arsenic. All chemicals, iron salt, ascorbic acid, potassium iodide, sodium borohydride were obtained from Merck. $\mathrm{pHs}$ were maintained using $0.1 \mathrm{~N} \mathrm{HNO}_{3}$ and $0.1 \mathrm{~N} \mathrm{NaOH}$. All studies were conducted using double distilled water. Analysis was carried out using AAS over mercury hydride system. The biochar was prepared using muffle furnace. The resulting biochar was used for arsenic removal.

\subsection{Method}

Biochar composite is developed using slow pyrolysis method in a muffle furnace. Biomasses were soaked in solution prepared from a known iron compound. The soaked biomasses in iron compound solution were then subjected to slow pyrolysis in muffle furnace under suitable thermal and temporal conditions. Iron biochar composite so obtained were washed, dried and used for conducting studies. $\mathrm{pH}$ optimization studies have been carried out in range 2-10. As(III) solution concentrations taken for conducting kinetic and isotherm studies ranged from 50-200 ug L ${ }^{-1}$. Characterization of material was carried over various instruments before and after arsenic adsorption to find the functional group, mineralogy, crystallinity (Table 1). Plausible mechanism for As(III) adsorption over biochar surface was thus established.

\section{RESULTS AND DISCUSSION}

\subsection{Characterization of adsorbents}

Both biochar composites were characterized for their surface area and porosity. High surface area and porosity was reported. Qualitative and quantitative analysis was done to find mineral composition, $\% \mathrm{C}, \mathrm{H}, \mathrm{N}$ and $\mathrm{S}$ content for both the biochar composites. Magnetic moment, oxidation states of various elemental species, mineralogy, crystallinity, elemental composition and functional groups were identified using PPMS, XPS, SEM, EDXRF, SEM-EDX, XRD, FTIR and ICP-MS (Fig. 1). Surface chemistry and hence the functional group identification made it possible to establish the mechanism involved in the adsorption of As(III) from water.

\subsection{Effect of initial $\mathrm{pH}$}

Sorption studies were performed in batch mode to obtain the equilibrium and kinetic constants and $\mathrm{pH}$ impact over arsenic adsorption from water on bio-char 


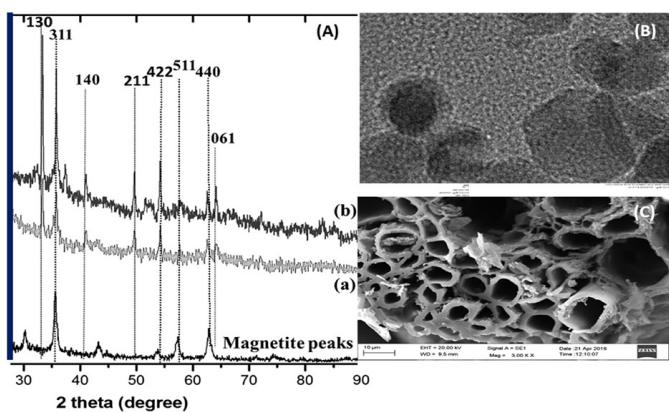

Figure 1. (A) XRD peaks (a) rice husk and (b) wheat husk biochar composite, (B) TEM image and (C) SEM image of biochar composites.

Table 1. Instruments used for adsorbent characterization.

\begin{tabular}{ll}
\hline $\begin{array}{l}\text { Characterization } \\
\text { techniques }\end{array}$ & Purpose \\
\hline $\begin{array}{l}\text { XPS } \\
\text { Quantachrome surface } \\
\text { area analyzer }\end{array}$ & $\begin{array}{l}\text { Oxidation states of elemental species } \\
\text { Specific surface area }\end{array}$ \\
SEM/EDX & Surface morphology \\
TEM & Crystallinity, magnetic domains \& stress \\
FT-IR & Functional group identification \\
FT-Raman & Compositional studies \\
XRD & Mineral composition \\
EDXRF & Elemental composition \\
PPMS & Magnetic moment \\
\hline
\end{tabular}

composites. Batch studies are considered because of their simplicity.

Preliminary results have shown good removal and efficiency so far. The maximum arsenic occurred in a $\mathrm{pH}$ range of 7.5-9.0 which is near to neutral $\mathrm{pH}$. This is advantageous as this will not require the adjustment of $\mathrm{pH}$ of water after arsenic removal.

\subsection{Adsorption kinetics and isotherm}

Kinetic studies were performed to examine the effect of adsorbent dose, contact time and temperature on arsenic adsorption rate. Pseudo second and first order rate equations were applied to establish adsorption kinetics and rate. Effect of adsorbent dose $\left(2 \mathrm{~g} \mathrm{~L}^{-1}\right.$, $1 \mathrm{~g} \mathrm{~L}^{-1}$ and $0.5 \mathrm{~g} \mathrm{~L}^{-1}$ ) on arsenic removal was studied. Kinetic experiments have showed good arsenic removal at low adsorbent dose. To establish mechanism and find the affinity between sorbate and sorbent, isotherm studies were conducted. Experimental values of adsorption capacities for rice husk and wheat husk biochar composites obtained were higher.

Adsorption data was modeled using Freundlich, Langmuir, Redlich-Peterson, Sips, Koble Corrigan, Radke-Prausnitz and Toth models to find various parameters necessary to design fixed bed reactors and find monolayer adsorption capacity. Information obtained from characterization has been applied to establish the probable mechanism for arsenic adsorption.

\subsection{Thermodynamic studies}

Thermodynamic studies were conducted to examine the adsorption behavior of arsenic onto the biochar surface. The parameters give the information about the feasibility and the spontaneity of the adsorption in terms whether adsorption process is endothermic or exothermic in nature. In following studies, the process was found to be endothermic in nature.

Desorption studies recover the toxic heavy metals from adsorbent for safe disposal as well as in keeping the process cost down.

\section{CONCLUSIONS}

Results from sorption studies have showed maximum removal of As(III). Kinetic have shown good adsorption capacity at lower optimum dose. Characterization studies provided information from which probable mechanisms, conditions, properties etc. of As(III) adsorption was established. This will now be utilized to conduct experiments with real groundwater samples to be collected from study area.

Here biochar is obtained from agricultural waste product that is very cost effective. These biochars can easily be used by developing modified biochar candles or columns to obtain arsenic free drinking water. Thus, composite biochars is considered to be a novel method for arsenic remediation. The biochar composites exhibited comparable sorption efficiency for arsenic removal. Therefore, this can easily replace commercial adsorbents.

\section{ACKNOWLEDGEMENTS}

One of the authors (PS) thanks to DST-INSPIRE for providing financial support to this work.

\section{REFERENCES}

Bhattacharya, P., Mukherjee, A. \& Mukherjee, A.B. 2011. Arsenic in groundwater of India. In: J.O. Nriagu (ed) Encyclopedia of Environmental Health, vol. 1. Burlington: Elsevier, pp. 150-164.

Hu, X., Ding, Z., Zimmerman, A.R., Wang, S. \& Gao, B. 2015. Batch and column sorption of arsenic onto iron impregnated biochar synthesized through hydrolysis. Water Res. 68: 206-216.

Ming, Z., Gao, B., Varnoosfaderani, S., Hebard, A., Yao, Y. \& Inyang, M. 2013. Preparation and characterization of a novel magnetic biochar for arsenic removal. Bioresour. Technol. 130: 4457-462.

Mohan, D. 2007. Arsenic removal from water/waste using adsorbents - a critical review. J. Hazard. Mater. 142(1-2): $1-53$.

Singh, S.K. 2015. Groundwater arsenic contamination in the Middle-Gangetic plain, Bihar (India): the danger arrived. Int. Res. J. Environ. Sc. 4(2): 70-76.

Sun, L., Chen, D., Shungang, W. \& Zebin. Y. 2015. Performance, kinetics and equilibrium of methylene blue adsorption on biochar derived from eucalyptus saw dust modified with citric, tartaric, and acetic acids. Bioresour. Technol. 198: 300-308. 